\title{
p16 and its putative interplay with metabolic factors in prostate cancer: An analysis based on public TCGA data
}

\author{
LAURA BOLDRINI $^{1}$, FRANCESCA MANASSERO ${ }^{2}$, CESARE SELLI $^{2}$, \\ RICCARDO BARTOLETTI $^{2}$ and PINUCCIA FAVIANA ${ }^{1}$
}

\author{
Departments of ${ }^{1}$ Surgical, Medical, Molecular Pathology and Critical Area, and ${ }^{2}$ Translational Research \\ and New Technologies in Medicine and Surgery, University of Pisa, I-56126 Pisa, Italy
}

Received November 8, 2019; Accepted January 16, 2020

DOI: $10.3892 /$ wasj.2020.33

\begin{abstract}
. p16 is one of the most common tumour suppressor genes, mainly due to its genetic inactivation. However, the clinical significance of $p 16$ in prostate cancer is not yet fully understood, and although p16 acts as a tumour suppressor gene, stress or oncogenic factors or alternative molecular events may overcome the role of p16 as a negative cell cycle regulator. p16 seems to be involved in the metabolic switch to glycolysis during tumorigenesis, possibly interacting with NADPH oxidase 4 (NOX4) and pyruvate kinase type M2 (PKM2), involved in energy metabolism, with differences depending on cell type. The aim of this study was to assess the putative crosstalk between p16, NOX4 and PKM2, with an involvement of miRNA-mediated regulation, in prostate cancer. Transcriptome data from a cohort of 243 patients were extracted from The Cancer Genome Atlas (TCGA) database. An elevated p16 expression level was significantly associated a high Gleason score, decreasing with the score $(\mathrm{P}<0.0001)$. NOX4 and PKM2 expression exhibited a similar trend as $p 16$, with higher values in the samples with Gleason scores of 9-10 samples $(\mathrm{P}<0.0001$ and $\mathrm{P}=0.02$, respectively). Moreover, bioinformatics analysis by TargetScan revealed that miR-625-5p could bind to the 3'UTR of $p 16$. A consequential pairing of the NOX4 and PKM 2 target region with miR-23a-3p and miR-122-5p, respectively was also found. Of note, the miR-625-5p levels inversely correlated with $p 16$ expression, miR-23a-3p and miR-122 with NOX4 and PKM2, respectively (data not shown). Taken together, these data suggest an interplay between p16 and metabolic factors, such as NOX4 and $P K M 2$, and a miRNA regulation, with a potential clinical
\end{abstract}

Correspondence to: Dr Laura Boldrini, Department of Surgical, Medical, Molecular Pathology and Critical Area, University of Pisa, Via Roma 57, I-56126 Pisa, Italy

E-mail: laura.boldrini@med.unipi.it

Key words: prostate cancer, p16, Gleason score, nicotinamide adenine dinucleotide phosphate oxidase 4, pyruvate kinase type $M 2$, miRNAs impact for the development of novel therapeutic strategies in prostate tumours.

\section{Introduction}

p16 is a 148 amino acid-protein encoded by the INK4a gene, which binds to cyclin-dependent kinases (CDKs) and through the inactivation of CDKs, induces growth arrest (1). pl6 is the second most common tumour suppressor gene, and the genetic inactivation due to missense mutations or promoter methylation of p16 itself is frequently found in cancer (2), namely in approximately $26 \%$ of all tumours (3). However, p16 mutations appear to be infrequent in prostate cancer (4); moreover, pl6 overexpression in cancer has not yet been fully clarified. Certain types of tumour, such as melanoma, HPV-associated tumours, non-small cell lung cancer, mesothelioma and lymphoma exhibit diminished p16 protein levels (5-7), while in other tumours, including prostate cancer, the overexpression of wild-type or mutant pl6 has been found $(8,9)$. pl6 overexpression is associated with tumour recurrence (10), and with a poor clinical course in patients with erythroblast transformation-specific-related gene (ERG)-negative prostate cancer (11); therefore, although p16 acts as a tumour suppressor gene, stress or oncogenic factors or alternative molecular events may overcome the role of p16 as a negative cell cycle regulator (12). Metabolic cellular reprogramming represents a key event in cancer cells, and p16 seems to be involved in the metabolic switch to glycolysis during tumorigenesis, also regulating NADPH Oxidase 4 (NOX4) expression in pancreatic ductal adenocarcinoma (13). Pyruvate Kinase type M2 (PKM2) also plays a central role in cancer, modulating glucose metabolism to support malignant cell proliferation (14). PKM2 expression has been in fact shown to be associated with tumour progression in several studies (15-19); however, its involvement in prostate cancer remains to be fully elucidated (20). Moreover, in recent years, microRNAs (miRNAs or miRs) have been evaluated as cancer regulators with a huge impact (21) on the management of several types of tumour, including prostate cancer.

This study attempted to assess the interaction between p16 and metabolic factors, such as NOX4 and PKM2, with a putative involvement of the miRNA-mediated regulation in prostate cancer. Thus, The Cancer Genome Atlas (TCGA) 
prostate cancer data were accessed, with the hypothesis that p16 plays a central role in prostate cancer progression, with an interaction with metabolic factors, as well as with miRNAs.

Taken together, p16, NOX4 and PKM2 expression were found to be decreased, possibly due to miR-625-5p, miR-23a-3p and miR-122-5p regulation, respectively, in cancer tissues with a low Gleason score, suggesting a deeper understanding of their interplay and of their regulation by miRNAs for developing novel therapeutic strategies for prostate tumours.

\section{Materials and methods}

TCGA database.IlluminaHiSeq expression data were extracted from the TCGA data portal (http://tcga.cancer.gov/; accessed October, 2017); expression data were downloaded along with the corresponding clinicopathological characteristics of 243 patients with prostate cancer.

Statistical analysis. One-way ANOVA and Tukey's test as a post hoc test were used for multiple comparisons. The Student's t-test was applied for comparisons of the mean expression values between 2 groups. Survival analyses were performed using the Kaplan-Meier method with the log-rank test for statistical significance. Using JMP10 software (SAS), a P-value $<0.05$ was considered to indicate a statistically significant difference.

miRNA regulation prediction analysis. Bioinformatic analysis was performed using the online database for miRNA target prediction, TargetScan (http://www.targetscan.org/), in order to reveal potential binding sites for miRNAs in the 3'UTR of the genes analysed in this study.

\section{Results}

TCGA database. A cohort of 243 patients with prostate cancer was extracted from the TCGA database. A lower Gleason score $(6,7)$ was observed in 144 out of the 243 TCGA cases, a Gleason score of 8 was observed in 27 cases, and a score of 9 in 71 cases; there was only 1 patient with a Gleason score of 10 . As regards tumour stage, there were 9 cases with stage T2a, 3 with T2b, 81 with T2c, 75 with T3a, 68 with T3b and 3 with T4 stage disease; in 4 cases, the T stage was unknown. The surgical margin resection status was $\mathrm{R} 0$ in 147 cases $(62.9 \%), \mathrm{R} 1$ in 75 cases (32\%), R2 in 4 cases $(1.7 \%)$ and RX in 8 cases $(3.4 \%)$; in 9 cases, the surgical margin resection status was unknown (Table I).

The disease free interval range was 0.76-165 months (median value, 34.4 months); the overall survival range was 0.76-165 months (median value, 37.8 months).

p16 expression and clinicopathological characteristics. An elevated p16 expression level was significantly associated with a high Gleason score, decreasing with the score $(\mathrm{P}<0.0001)$; the mean value was 886,646 in cases with a Gleason score of 9-10, 558,832 in those with a score of 7-8 and 472,610 in cases with a score of 6 . When comparing tumour stage T2 versus T3/T4, it was found that high p16 levels were associated with an advanced tumour stage (t-test; $\mathrm{P}=0.001)$, as well
Table I. Clinicopathological characteristics of the 243 prostate cancer patients extracted from the TCGA database.

\begin{tabular}{lc}
\hline Characteristics & No. of samples \\
\hline Gleason score & 144 \\
$6-7$ & 27 \\
8 & 72 \\
$9-10$ & \\
T stage & 9 \\
T2a & 3 \\
T2b & 81 \\
T2c & 75 \\
T3a & 68 \\
T3b & 3 \\
T4 & 4 \\
Unknown & \\
Surgical margin status & \\
R0 & 147 \\
R1 & 75 \\
R2 & 4 \\
RX & 8 \\
Unknown & 9 \\
\hline
\end{tabular}

as with a positive surgical resection margins status $\mathrm{R} 1 / \mathrm{R} 2$ in comparison to R0 (t-test; $\mathrm{P}=0.005$ ) (Fig. 1).

NOX4 expression. The samples with a Gleason of 9-10 exhibited a higher value of $N O X 4$ expression $(664,737 \pm 59,395)$, those with a score of 6 exhibited the lowest $(20,7001 \pm 102,158)$, and those with a score of $7-8(333,929 \pm 41,275)$ exhibited intermediate and increasing values $(\mathrm{P}<0.0001)$ (Fig. 2). pl6 and NOX4 expression were positively associated $(\mathrm{P}<0.0001)$ (Fig. 3).

PKM2 expression. PKM2 expression exhibited a similar trend $(\mathrm{P}=0.02)$, as that of $p 16$; the samples with a Gleason score of 9-10 exhibited a higher value of PKM2 expression, while a lower $P K M 2$ value was found in the prostate tumours with a score of 6 (Fig. 4). A high pl6 expression was significantly associated with elevated $P K M 2$ levels $(\mathrm{P}<0.0001)$ (Fig. 5); moreover, as shown in Fig. 6, a positive association was observed between PKM2 and NOX4 ( $\mathrm{P}=0.0006)$.

miRNA regulation. Bioinformatics analysis using TargetScan revealed that miR-625-5p could bind to the 3'UTR of $p 16$. In addition, a consequential pairing of the NOX4 and PKM2 target region with $\mathrm{miR}-23 \mathrm{a}-3 \mathrm{p}$ and $\mathrm{miR}-122-5 \mathrm{p}$, respectively was found. The predicted pairing with miRNAs is illustrated in Fig. 7. Of note, the miR-625-5p levels were inversely correlated with $p 16$ expression, and miR-23a-3p and miR-122-5p correlated with $N O X 4$ and $P K M 2$, respectively (data not shown).

p16 expression and survival. As regards patient survival, as shown in Fig. 8, Kaplan-Meier analysis revealed a shorter 

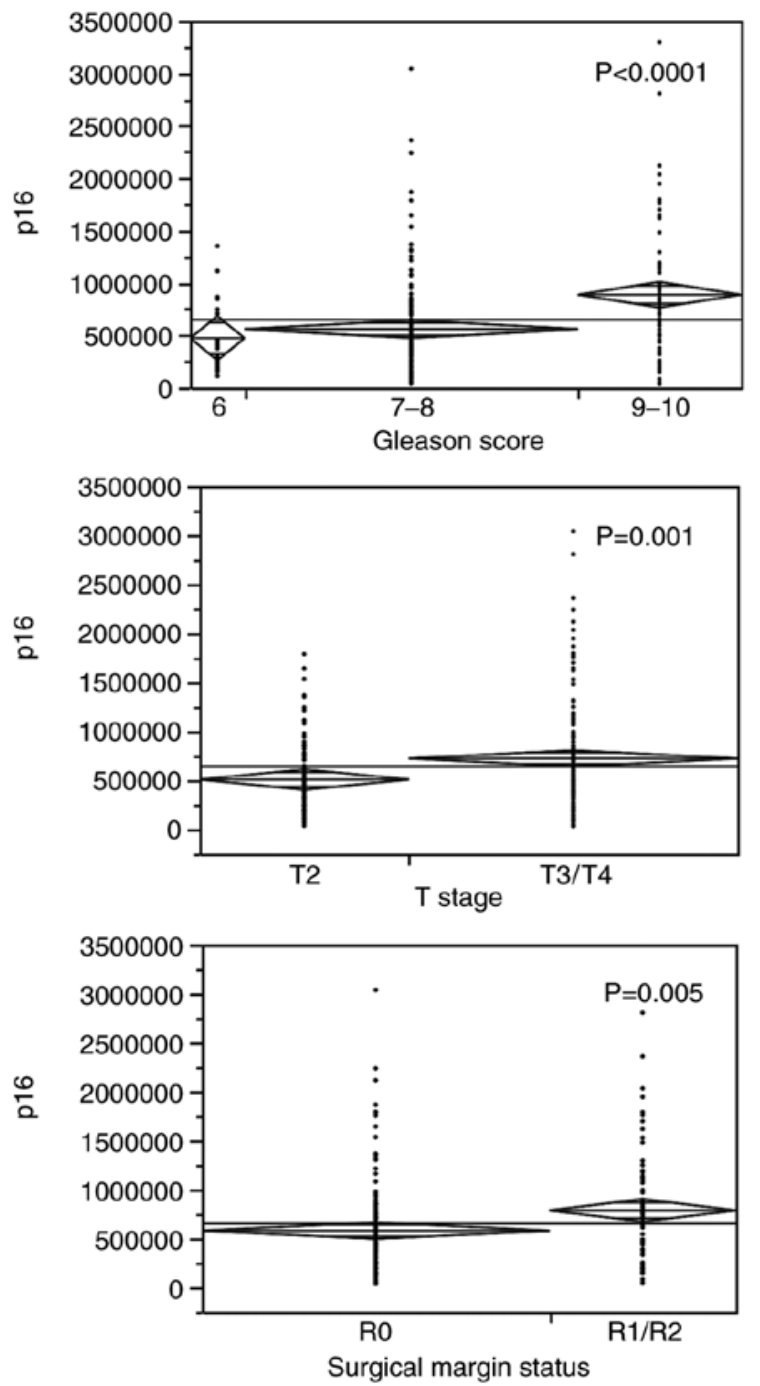

Figure 1. Association between the p16 expression level and Gleason score (upper panel), tumour stage $\mathrm{T}$ (middle panel) and surgical margin status $\mathrm{R}$ (lower panel) in 243 prostate cancer samples from the TCGA database. TCGA, The Cancer Genome Atlas.

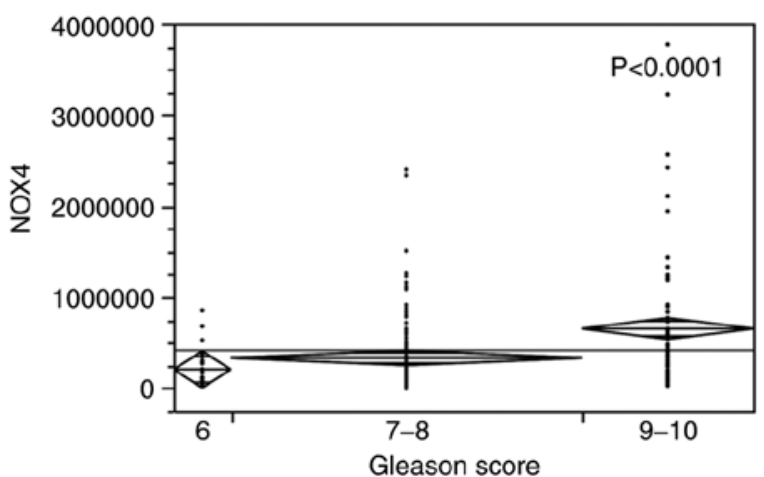

Figure 2. Association between the NOX4 expression level and Gleason score in 243 prostate cancer samples from the TCGA database. NOX4, NADPH oxidase 4. Nox4, NADPH oxidase 4; NADPH, Nicotinamide adenine dinucleotide phosphate.

disease-free or overall survival in patients with a high $p 16$ expression ( $28.25 \pm 2.38$ months and $30.85 \pm 2.44$, respectively) in comparison to those with low p16 levels $(30.98 \pm 2.33$ and $36.01 \pm 2.36$ ), although no statistically significant differences

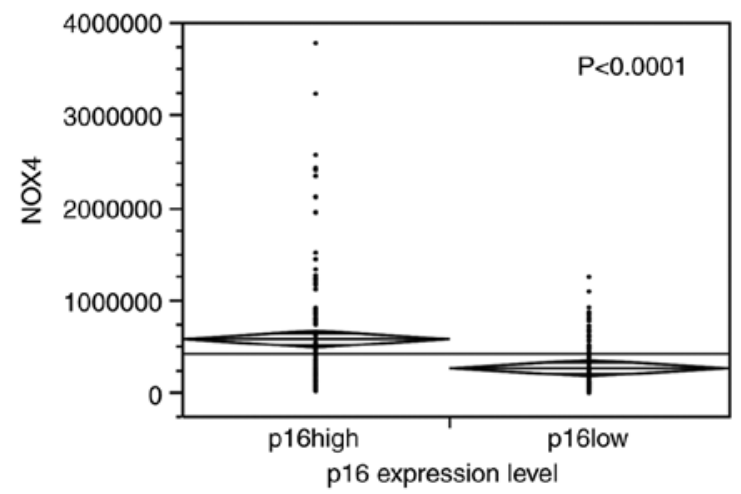

Figure 3. Association between $p 16$ and NOX4 expression in 243 prostate cancer samples from the TCGA database. NOX4, NADPH oxidase 4 .

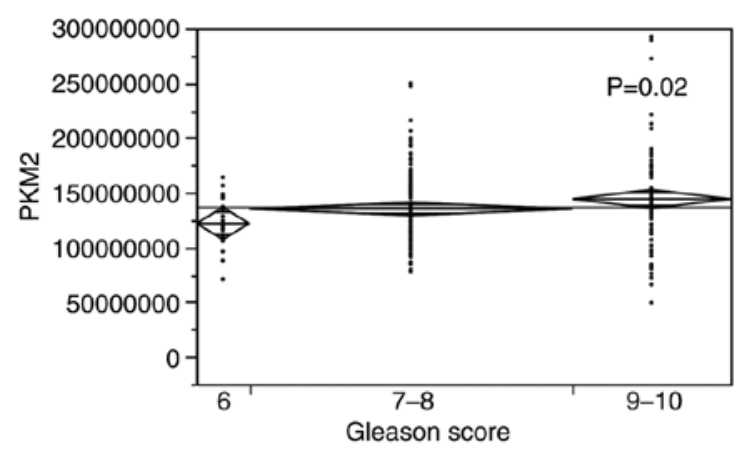

Figure 4. Association between the PKM2 expression level and Gleason score in 243 prostate cancer samples from the TCGA database. PKM2, pyruvate kinase M2.

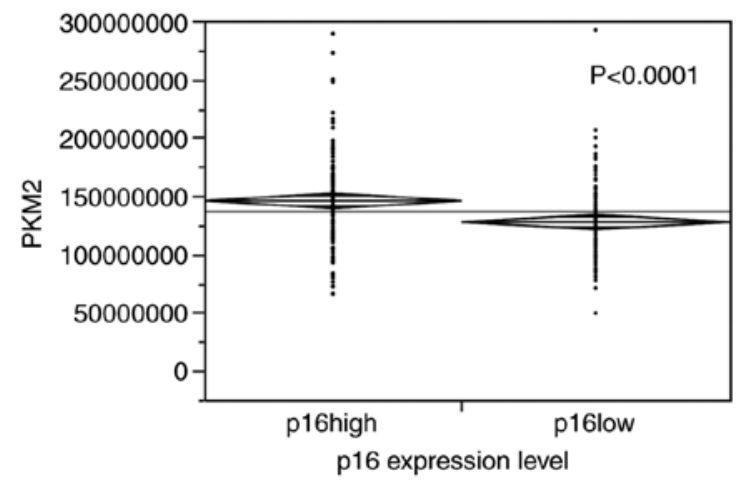

Figure 5. Association between $p 16$ and $P K M 2$ expression in 243 prostate cancer samples from the TCGA database. PKM2, pyruvate kinase M2.

were observed $(\mathrm{P}=0.11$ and $\mathrm{P}=0.31$, for disease-free interval and overall survival, respectively); this may be due to the absolute excellent survival rate in prostate cancer.

\section{Discussion}

The exact role of p16 in prostate cancer progression has not yet been fully determined. p16 is one of the proteins which has been most extensively studied over the past three decades, with its high expression being associated with a more aggressive clinical development in several neoplastic diseases, such as melanoma, lymphoma and non-small cell lung cancer $(5-7,22)$. 


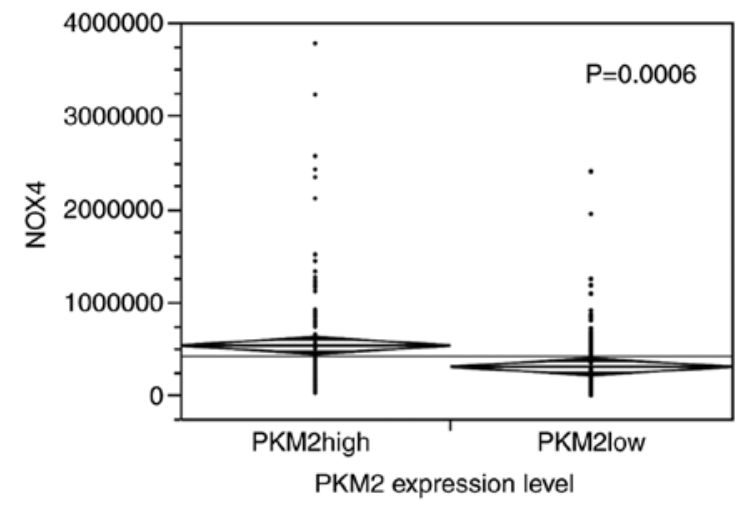

Figure 6. Association between $P K M 2$ and NOX4 expression in 243 prostate cancer samples from the TCGA database. $P K M 2$, pyruvate kinase M2; NOX4, NADPH oxidase 4.

A

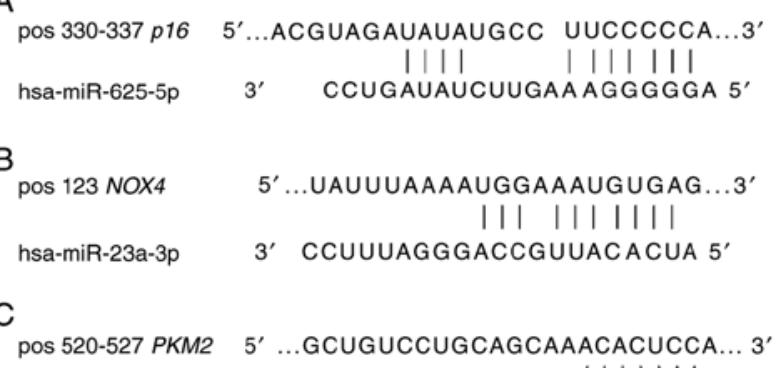

Figure 7. (A) Predicted consequential pairing, shown in bold, of the p16 target region (top panel) and miR-625-5p (bottom panel). (B) Predicted consequential pairing, shown in bold, of the NOX4 target region (top panel) and miR-23a-3p (bottom panel). (C) Predicted consequential pairing, shown in bold, of the PKM2 target region (top panel) and miR-122-5p (bottom panel). Predicted consequential pairing was carried out using TargetScan (http://www.targetscan.org/). NOX4, NADPH oxidase 4; PKM2, pyruvate kinase M2.

On the contrary, recently, patients with prostate cancer with p16 overexpression were shown to have a more aggressive behaviour (11). The overexpression of wild-type or mutant pl6 may involve an effort to recover p16 functions in certain types of tumour; however, the mechanisms responsible for the development and progression of prostate cancer remain unknown. pl6 dysregulation has been shown to be involved in metabolic reprogramming in pancreatic cancer cells (13), inducing NOX4 activity, one of the NADPH oxidases required to maintain glycolysis for tumoural cell growth. Recently, NOX4 silencing has been suggested as a theoretical target in prostate cancer, considering the effect of repressed glycolysis, with decreased lactate and ATP production, on cell proliferation (23). PKM2 is another well-established regulator of glycolysis and energy metabolism of cancer cells (24). Apart from its role in aerobic glycolysis, PKM2 seems to be also involved in non-metabolic functions, such as cell cycle progression; however, the mechanisms underlying its regulation in tumour progression are not yet clear, and this may be dependent on the cancer cell type (25).

This study accessed the public TCGA database in order to clarify the role of $p 16$ in prostate cancer and its putative
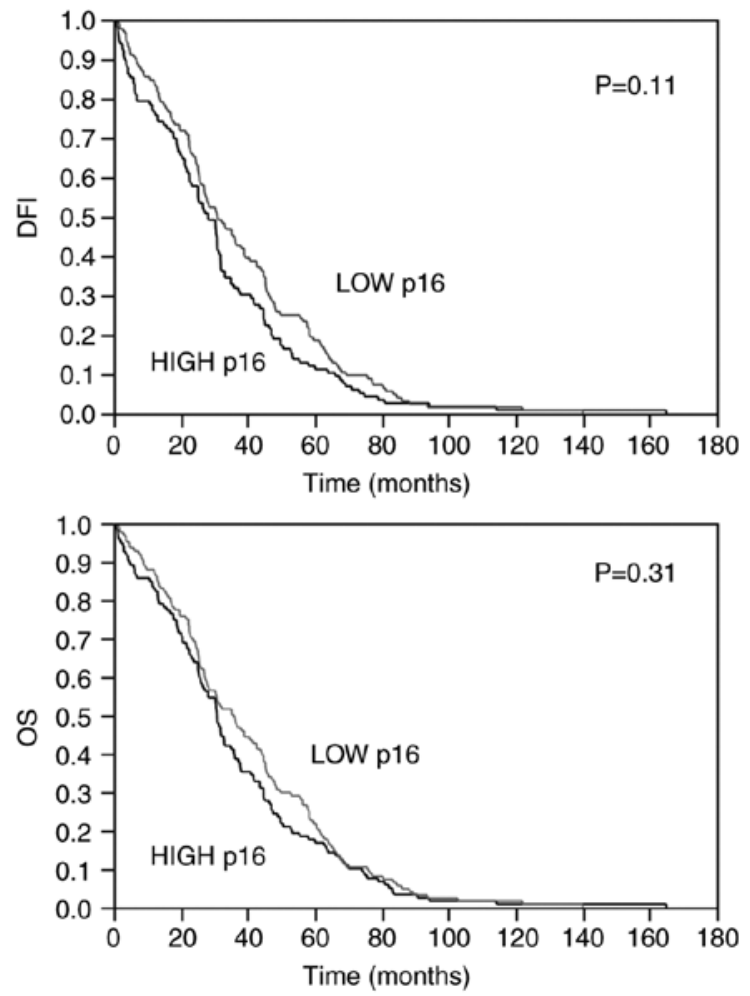

Figure 8. Kaplan-Meier curves for survival analysis [disease-free interval (DFI; upper panel) and overall survival (OS; lower panel)] in 243 prostate cancer samples with a low p16 (grey line) and high p16 expression level (black line).

interaction with $N O X 4$ and $P K M 2$; a possible miRNA regulation was also analysed. It was found that the p16 expression level was increased in samples with a higher Gleason score and in those with an advanced tumour stage. It was also associated with positive surgical resection margins and with a trend to a worse survival, confirming the involvement of pl6 in the biological switch to advanced prostate cancer. $p 16$ upregulation was linked to either a NOX4 or PKM2 high expression level, suggesting a putative network linking p16 and metabolic genes in modulating growth control and prostate cancer progression. The role of NOX4 and PKM2 in reprogramming the p16-induced metabolic switch to glycolysis may identify a novel therapeutic target for prostate tumours.

miRNAs are small, non-coding molecules involved in repressing translation by binding the 3'UTR of their target genes (26). In recent years, several studies have demonstrated the role of miRNAs in diagnosis, prognosis and as predictive biomarkers in different cancer types (27-30). In this study, bioinformatics analysis using TargetScan revealed that either p16 or NOX4 and PKM2 3'UTR could bind to miR-625-5p, miR-23a-3p and miR-122-5p, respectively, with downregulation due to consequential pairing, suggesting the three genes as physiologically targets of corresponding miRNAs.

pl6 may be involved in overlapping pathways; its contribution as a tumour suppressor gene has been well-established, while the particular role of p16 deregulation to the development and progression of a specific tumour remain to be further explored. A complex coordination of pl6 with other molecular events occurring in the same tumour microenvironment may explain this intricate role of $p 16$. 
Taken together, the data of this study suggest interplay of p16 with modulating metabolism markers, with a regulation by miRNAs in prostate tumours; uncovering the putative regulatory network of p16 may have a potential clinical impact on the development of novel therapeutic strategies for prostate cancer.

\section{Acknowledgements}

Not applicable.

\section{Funding}

No funding was received.

\section{Availability of data and materials}

The data supporting the conclusions of this article are available from the corresponding author on reasonable request.

\section{Authors' contributions}

All the authors (LB, FM, CS, RB and PF) conceived the study. $\mathrm{LB}$ and $\mathrm{PF}$ were involved in data collection, project development and management, manuscript writing and editing. All authors have read and approved the final manuscript.

\section{Ethics approval and consent to participate}

Not applicable.

\section{Patient consent for publication}

Not applicable.

\section{Competing interests}

The authors declare that they have no competing interests.

\section{References}

1. Serrano M, Hannon GJ and Beach D: A new regulatory motif in cell cycle control causing specific inhibition of cyclinD-cdk4. Nature 366: 704-707, 1993.

2. Serrano M: The tumor suppressor protein p16INK4a. Exp Cell Res 237: 7-13, 1997.

3. Liggett WH and Sidransky D: Role of the p16 tumor suppressor gene in cancer. J Clin Oncol 16: 1197-1206, 1998.

4. Tamimi Y, Bringuier PP, Smit F, van Bokhoven A, Debruyne FM and Schalken JA: p16 mutations/deletions are not frequent events in prostate cancer. Brit J Cancer 74: 120-122, 1996.

5. Taga S, Osaki T, Ohgami A, Imoto H, Yoshimatsu T, Yoshino I, Yano K, Nakanishi R, Ichiyoshi Y and Yasumoto K: Prognostic value of the immunohistochemical detection of p16INK4 expression in non small cell lung carcinoma. Cancer 80: 389-395, 1997.

6. García-Sanz R, González M, Vargas M, Chillón MC, Balanzategui A, Barbón M, Flores MT and San Miguel JF: Deletions and rearrangements of cyclin-dependent kinase 4 inhibitor gene p16 are associated with poor prognosis in B cell non-Hodgkin's lymphomas. Leukemia 11: 1915-1920, 1997.

7. Hwang HC, Sheffield BS, Rodriguez S, Thompson K, Tse $\mathrm{CH}$, Gown AM and Churg A: Utility of BAP1 immunohistochemistry and p16 (CDKN2A) FISH in the diagnosis of malignant mesothelioma in effusion cytology specimens. Am J Surg Pathol 40: 120-126, 2016.

8. Mahajan A: Practical issues in the application of p16 immunohistochemistry in diagnostic pathology. Hum Pathol 51: 64-74, 2016.

9. Serra S and Chetty R: p16. J Clin Pathol 71: 853-858, 2018.
10. Lee CT, Capodieci P, Osman I, Fazzari M, Ferrara J, Scher HI and Cordon-Cardo C: Overexpression of the cyclin-dependent kinase inhibitor p16 is associated with tumor recurrence in human prostate cancer. Clin Cancer Res 5: 977-983, 1999.

11. Burdelski C, Dieckmann T, Heumann A, Hube-Magg C, Kluth M, Beyer B, Steuber T, Pompe R, Graefen M, Simon R, et al: p16 upregulation is linked to poor prognosis in ERG negative prostate cancer. Tumour Biol 37: 12655-12663, 2016

12. $\mathrm{Li} \mathrm{J}$, Poi MJ and Tsai MD: Regulatory mechanisms of tumor suppressor P16(INK4A) and their relevance to cancer. Biochemistry 50: 5566-5582, 2011.

13. Ju HQ, Ying H, Tian T, Ling J, Fu J, Lu Y, Wu M, Yang L, Achreja A, Chen G, et al: Mutant Kras- and p16-regulated NOX4 activation overcomes metabolic checkpoints in development of pancreatic ductal adenocarcinoma. Nat Commun 8: 14437, 2017.

14. Zhu $\mathrm{H}$, Luo $\mathrm{H}$, Zhu X, Hu X, Zheng L and Zhu X: Pyruvate kinase M2 (PKM2) expression correlates with prognosis in solid cancers: A meta-analysis. Oncotarget 8: 1628-1640, 2017.

15. Goldberg MS and Sharp PA: Pyruvate kinase M2-specific siRNA induces apoptosis and tumor regression. J Exp Med 209: 217-224, 2012.

16. Hsu MC, Hung WC, Yamaguchi H, Lim SO, Liao HW, Tsai CH and Hung MC: Extracellular PKM2 induces cancer proliferation by activating the EGFR signalling pathway. Am J Cancer Res 6 : 628-638, 2016.

17. Singh H, Longo DL and Chabner BA: Improving prospects for targeting RAS. J Clin Oncol 33: 3650-3659, 2015.

18. Sun Q, Chen X, Ma J, Peng H, Wang F, Zha X, Wang Y, Jing Y, Yang $\mathrm{H}$, Chen $\mathrm{R}$, et al: Mammalian target of rapamycin up-regulation of pyruvate kinase isoenzyme type M2 is critical for aerobic glycolysis and tumor growth. Proc Natl Acad Sci USA 108: 4129-4134, 2011.

19. Yang W, Xia Y, Ji H, Zheng Y, Liang J, Huang W, Gao X, Aldape K and Lu Z: Nuclear PKM2 regulates beta-catenin transactivation upon EGFR activation. Nature 480: 118-122, 2011.

20. Wong N, Yan J, Ojo D, De Melo J, Cutz JC and Tang D: Changes in PKM2 associate with prostate cancer progression. Cancer Invest 32: 330-338, 2014.

21. Iorio MV and Croce CM: MicroRNAs in cancer: Small molecules with a huge impact. J Clin Oncol 27: 5848-5856, 2009.

22. Straume O and Akslen L: Alterations and prognostic significance of p16 and p53 protein expression in subgroups of cutaneous melanoma. Int J Cancer 74: 535-539, 1997.

23. Wu QQ, Zheng B, Weng GB, Yang HM, Ren Y, Weng XJ, Zhang SW and Zhu WZ: Downregulated NOX4 underlies a novel inhibitory role of microRNA-137 in prostate cancer. J Cell Biochem 120: 10215-10227, 2019.

24. Yoshida GJ: Metabolic reprogramming: The emerging concept and associated therapeutic strategies. J Exp Clin Cancer Res 34: $111,2015$.

25. Dey P, Kundu A, Sachan R, Park JH, Ahn MY, Yoon K, Lee J, Kim ND, Kim IS, Lee BM and Kim HS: PKM2 knockdown induces autophagic cell death via AKT/mTOR pathway in human prostate cancer cells. Cell Physiol Biochem 52: 1535-1552, 2019.

26. Etheridge A, Lee I, Hood L, Galas D and Wang K: Extracellular microRNA: A new source of biomarkers. Mutat Res 717: 85-90, 2011.

27. Volinia S and Croce CM: Prognostic microRNA/mRNA signature from the integrated analysis of patients with invasive breast cancer. Proc Natl Acad Sci USA 110: 7413-7417, 2013

28. Feber A, Xi L, Pennathur A, Gooding WE, Bandia S, Wu M, Luketich JD, Godfrey TE and Litle VR: MicroRNA prognostic signature for nodal metastases and survival in esophageal adenocarcinoma. Ann Thorac Surg 91: 1523-1530, 2011.

29. Beer DG, Kardia SL, Huang C, Giordano TJ, Levin AM, Misek DE, Lin L, Chen G, Gharib TG, Thomas DG, et al: Gene-expression profiles predict survival of patients with lung adenocarcinoma. Nat Med 8: 816-824, 2002.

30. Shukla S, Evans JR, Malik R, Feng FY, Dhanasekaran SM, Cao X, Chen G, Beer DG, Jiang H and Chinnaiyan AM: Development of a RNA-Seq based prognostic signature in lung adenocarcinoma. J Natl Cancer Inst 109, 2016.

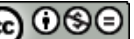

This work is licensed under a Creative Commons Attribution-NonCommercial-NoDerivatives 4.0 International (CC BY-NC-ND 4.0) License. 